\title{
Morbidity, cost and role of health care worker transmission in an influenza outbreak in a tertiary care hospital
}

ANNALEE YASSI, MD, MSC, FRCPC, MYRNA MCGILL, RN, COHN, DONNA HOLTON, MD, FRCPC, LINDSAY NICOLLE, MD, FRCPC

\begin{abstract}
A YASSi, M MCGLl, D Holton, L Nicolle. Morbidity, cost and role of health care worker transmission in an influenza outbreak in a tertiary care hospital. Can J Infect Dis 1993;4(1):52-56. An influenza A outbreak involving 17 health care workers (HCWs) and 16 chronic geriatric patients on a ward in a tertiary care hospital was reviewed. Thirty-seven per cent of all HCWs and $47 \%$ of patients on the affected wards became ill with influenza. Three patients died during the outbreak. The majority of health care workers became ill prior to detecting the first patient case of influenza, suggesting that nosocomial spread from HCWs to patients may have occurred. Only $13.7 \%$ of the staff and $5.9 \%$ of the patients had been vaccinated prior to the outbreak. Lost time due to $\mathrm{HCW}$ absenteeism, outbreak-related medication costs and additional staff time involved in outbreak control resulted in considerable cost to the hospital. It is suggested that much of this cost, as well as morbidity and possibly mortality, could have been avoided by increased immunization of HCWs and patients.
\end{abstract}

Key Words: Cost, Health care worker, Influenza outbreak, Nosocomial transmission

\section{Morbidité, coût et rôle de la contagion parmi des travailleurs de la santé dans un centre hospitalier de soins tertiaires touché par une épidémie d'influenza}

RÉSUMÉ: Une épidémie d'influenza A, touchant 17 travailleurs de la santé et 16 patients gériatriques chroniques dans un service d'un centre hospitalier de soins tertiaires a été analysée. Trente-sept pour cent de ces travailleurs de la santé, et $47 \%$ des patients des unités affectées ont présenté un influenza. Trois patients sont décédês durant l'épidémie. La majorité des travailleurs de la santé sont devenus malades avant que le premier cas d'influenza ne soit déclaré chez les patients, ce qui donne à penser qu'il y a eu propagation nosocomiale des travailleurs de la santé vers leurs bénéficiaires. Seulement $13.7 \%$ du personnel et $5.9 \%$ des patients avaient été vaccinés avant l'épidémie. La perte de productivité due à l'absentéisme, le coût des médicaments associés à l'épidémie et le recours aux heures supplémentaires qu'a occasionnés la situation a imposé des dépenses considérables à l'établissement. Il est fort probable qu'une bonne part de ces coûts, de même que la morbidité et possiblement la mortalité auraient pu ètre évitées par une immunisation plus généralisée des travailleurs de la santé et des patients.

Department of Community Health Sciences and Medicine, University of Manitoba and Health Sciences Centre, Winnipeg, Manitoba

Correspondence and reprints: Dr A Yassi, Director, Occupational and Environmental Health Program, Department of Community Health Sciences and Medicine, University of Manitoba, 820 Sherbrook Street, Winnipeg. Manitoba R3A 1R9

Received for publication June 14, 1991. Accepted April 16, 1992 
E ACH WINTER, INFLUENZA VIRUSES CAUSE A DRAMATIC INcrease in visits to physicians' offices and hospital emergency rooms across Canada (1-9). Influenza outbreaks are also known to result in increased absenteeism and lost productivity among hospital staff (9-11). Descriptive reports of hospital influenza outbreaks provide evidence that nosocomial spread of influenza occurs (6-9). It generally is believed, although it has never been unequivocally documented, that hospital staff can transmit the infection to patients (9). The role of hospital staff in the transmission and perpetuation of influenza outbreaks, and the financial impact of such outbreaks on the hospital have never been the focus of a study. This paper describes the involvement of health care workers (HCWs) and the impact of an influenza A outbreak in the geriatric wards of a tertiary care hospital.

Influenza in elderly patients and in those with underlying cardiopulmonary abnormalities frequently is accompanied by lower respiratory tract complications and death (1,2,12-14). Risk factors for influenza A acquisition in institutionalized elderly patients are considerable. Use of influenza vaccination and amantadine prophylaxis to prevent disease in high risk groups is effective; however, only $20 \%$ of eligible high risk outpatients are vaccinated $(1,2,9,15)$. The reported rate of vaccination in institutional settings has varied from 0 to $97 \%$ (16).

\section{OUTBREAK DESCRIPTION}

On December 4, 1989, an outbreak of acute respiratory disease was detected on a geriatric ward at the Health Sciences Centre in Winnipeg, Manitoba. Sixteen of 34 patients (47\%) became ill during the outbreak. After the isolate was confirmed to be influenza A, influenza vaccination status was determined for all patients on the ward. The infection control team recommended that all well patients not previously vaccinated and who were not allergic to eggs (13 patients) be vaccinated and given amantadine prophylaxis for two weeks. A five-day course of amantadine was recommended for the one patient who had been ill for less than $48 \mathrm{~h}$. Amantadine prophylaxis and vaccination were also recommended for all new admissions to the ward who had not been vaccinated for the 1989 season. The dose of amantadine was decreased from $100 \mathrm{mg}$ daily to $100 \mathrm{mg}$ on alternate days if the patient had an elevated creatinine level or history of seizures or cerebral vascular accidents. Creatine level was also determined after completion of amantadine therapy.

Fifty-one HCWs provided care for the hospital's two geriatric wards. While 22 of these HCWs did not work primarily on the geriatric ward which housed the 16 patients with influenza-like illness, they were included in the outbreak investigation due to considerable crossassigning of staff between the two wards. Occupational health nurses interviewed staff and provided influenza vaccine and amantadine to HCWs who had not been previously vaccinated for the 1989 season. Both occupational health and infection control nurses provided inservice information about influenza A.

\section{PATIENTS AND METHODS}

Data on morbidity and absenteeism in HCWs were collected from questionnaires administered by the occupational health nurses in the course of outbreak control procedures. A HCW was considered to have had influenza by the occupational health team if there was a history of fever and at least two 'flu-related symptoms, including headache, cough (productive or nonproductive), myalgia, arthralgia, malaise or sore throat. As cases were defined retrospectively - sometimes as much as three weeks later - no confirming serology was drawn.

Records of patients residing on the ward from November 20, 1989, until December 20, 1989, were reviewed by the infection control team using ward census data. Patients with respiratory symptoms (rhinitis, conjunctivitis, sore throat, productive or nonproductive cough) or fever (more than $37.5^{\circ} \mathrm{C}$ ) and malaise/ prostration were considered clinical cases if no other foci of infection were identified. Cases were defined as confirmed if a throat or sputum culture grew influenza A or if a fourfold rise in acute versus convalescent influenza A serology was found using complement fixation (Virion US Incorporated, Institute Virion AG, Rueschliken-Zuerich, Switzerland). A case control study was performed to determine risk factors for disease acquisition. Eight patients who shared a room with a case but who did not become ill were identified.

Statistical analysis of risk factors was performed using $\chi^{2}$, Fischer's exact test and Student's $t$ test.

Costs of the outbreak included in this analysis were restricted to time lost by ward staff for attending outbreak control activities, ward staff absenteeism due to influenza illness, cost of medication and occupational health nurses' time related directly to outbreak management activities, such as brief health assessment, screening for the need for vaccination and/or amantadine, as well as provision of other advice regarding infection control follow-up. Physicians' time for outbreak control activities were not included because no direct increased costs ensued. The cost of the vaccine was also excluded as these costs would have occurred had the HCWs been vaccinated prior to the influenza season. For the same reason, occupational health nurses' time related to providing information, seeking consent and vaccinating the HCWs were excluded from total outbreak activity time. Additional ward nursing time generated by the complications in ward patients was not quantifiable as no additional staff were brought in for this purpose. 


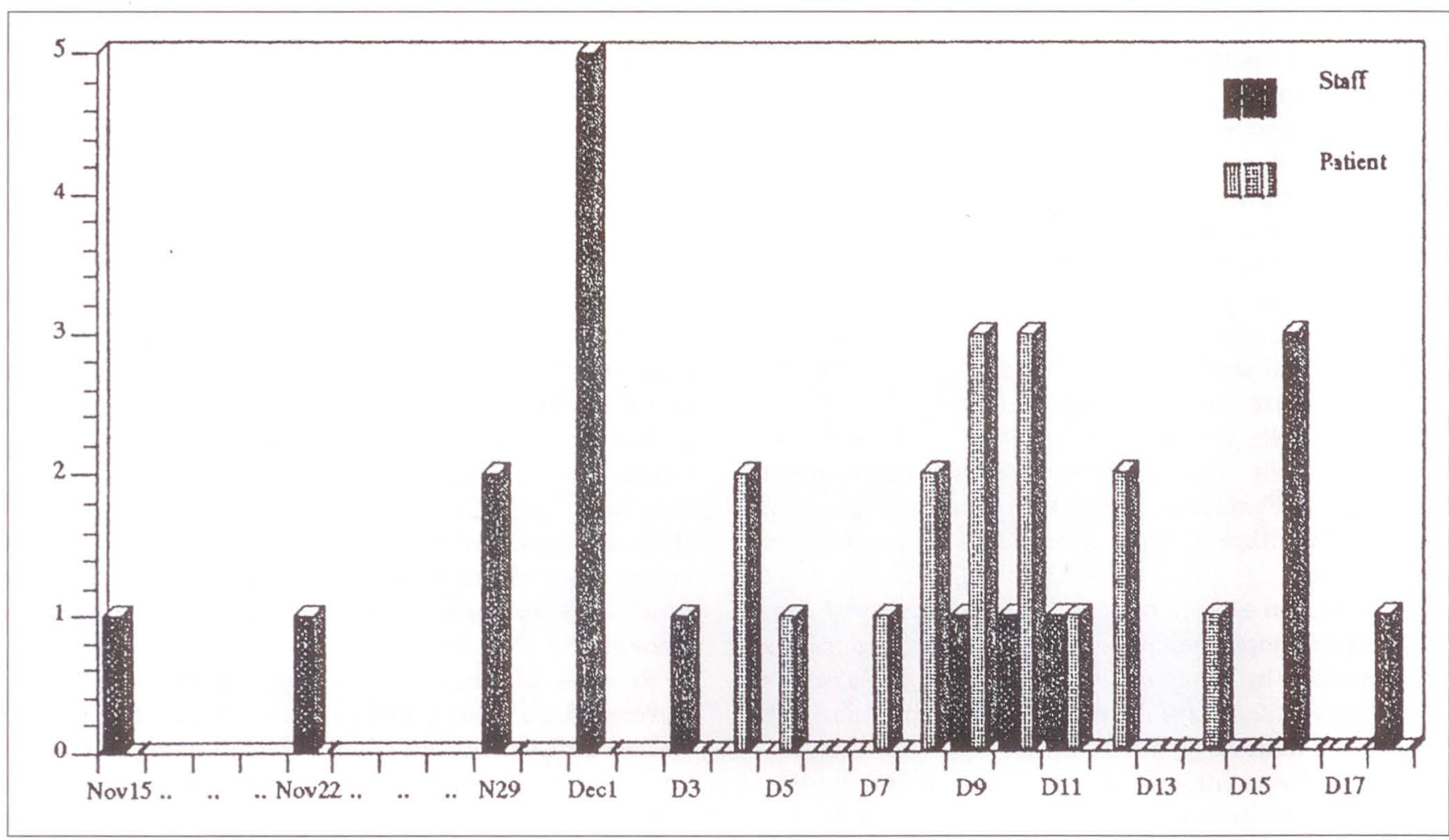

Figure 1) Staff and patient morbidity by date of influenza A onset

\section{RESULTS}

A respiratory illness was documented in 19 of the 51 HCWs (37.3\%) and 16 of 34 patients (47\%) from December 4 to 14,1989 . Twelve patients were confirmed as cases; four patients had a positive throat culture, one had a positive sputum culture, and 10 demonstrated a fourfold rise in acute and convalescent influenza A serology. Laboratory data were not obtained from the four persons classified as clinical cases.

The mean age of the patients was 84.8 years (range 72 to 96$)$. Seven of 16 patients (43.8\%) had nausea, six $(37.5 \%)$ had malaise and $14(87.5 \%)$ had cough (11 had a dry cough, three had a productive cough). Nine of 14 patients $(64.2 \%)$ had fever while one of 11 patients (9.1\%) had leukocytosis. Three patients, all clinical cases identified retrospectively, died during the outbreak. A respiratory illness was documented as a potential cause of death in each instance.

Eight of the initial nine patients who became ill had documented history of congestive heart failure. In contrast, only one of the subsequent seven patients who became ill had a history of that disease. No ill patients shared a room during the first six days of the epidemic. Previous history of congestive heart failure or the current use of furosemide was a significant risk factor when eight pairs of cases and controls who shared a room were compared (odds ratio $21,95 \%$ confidence interval: 1.09 to $1099, \mathrm{P}=0.04$, Fischer's exact test). No significant differences in age, sex, presence of chronic obstructive lung disease, diabetes mellitus, hyperten- sion, myocardial infarction, renal disease or use of digoxin between cases and controls were documented.

Two of 16 patient cases $(12.5 \%)$ received amantadine treatment and 13 of 18 eligible patients (72.2\%) received amantadine prophylaxis. No adverse effects occurred among patients administered amantadine. No change in creatinine was observed in any of the 11 patients who received amantadine prophylaxis. Three patients did not have post amantadine administration creatinine level determined.

Figure 1 indicates the date of onset of influenza symptoms in patients and HCWs fulfilling the criteria for inclusion. Only 16 of $19 \mathrm{HCWs}$ noted a date of onset of illness on their questionnaires. As shown in Figure 1 , nine of these $16 \mathrm{HCWs}$ reported illness prior to any patient becoming ill. The peaks of the outbreak occurred on December 1 and from December 9 to 10 for HCWs and patients, respectively. The staff had frequent and direct contact with these geriatric patients during nursing care. These patients were all long term inpatients who had minimal contact with the general public, although they did receive visits from family and friends, and were encouraged to attend a common dining room for meals and to participate in group activities. None of the ill patients was known to have been visited by someone with a respiratory illness. It was also noted that seven of the 16 staff developed influenza after the outbreak was detected in patients.

Only seven of the 51 HCWs (13.7\%) had been vaccinated prior to the outbreak (Table 1). Of the 
remainder, 41 staff received vaccine during the outbreak. Three HCWs were not vaccinated, due either to pregnancy or allergy to eggs. Only three patients (one control and two cases) had received influenza vaccination prior to the outbreak. Thirteen of 17 eligible well patients $(76.5 \%)$ were vaccinated during the outbreak. Four patients became confirmed influenza cases within two days of vaccination.

Twelve of the 19 staff with influenza $(63.2 \%)$ were absent for at least one day ( $7.5 \mathrm{~h}$ of work time). A total of $165 \mathrm{~h}$ of work time was lost due to influenza-related absenteeism. At $\$ 15.71 / \mathrm{h}$, the average cost of wages and benefits for these workers resulted in a direct cost of $\$ 2,592.15$. Ward staff also lost work time attending the outbreak follow-up program (approximately 20 mins per person or \$267.07). Approximately $35 \mathrm{~h}$ of additional occupational health nurses' time was spent on special outbreak activities. At a cost of $\$ 17.60 / \mathrm{h}$, this resulted in an additional cost of $\$ 616.00$. Physicians from occupational medicine, as well as infection control, were involved in follow-up of the outbreak. The physicians saw sick patients or staff who would not normally have been seen by them; they prescribed medications and/or wrote orders for patients with influenza-related complications. During the outbreak, the attending physicians prescribed antibiotics for 10 of 16 patient cases (62.5\%). The antibiotics cost $\$ 526.00$, excluding the cost of intravenous administration sets, and the additional nursing and pharmacy costs associated with the antibiotic prescribed. The cost of amantadine for the outbreak was $\$ 607.00$. Thus, the immediate and easily quantifiable direct costs were approximately $\$ 5,000.00$. Indirect costs were likely considerably higher.

\section{DISCUSSION}

This influenza outbreak affected a large proportion of geriatric patients and the HCWs who cared for them (47 and 37\%, respectively). While viral cultures and serologies were not obtained for all patients or HCWs, no other agent was known to be prevalent at the time. The fact that patients became ill only after nine HCWs developed influenza symptoms and the fact that these HCWs had direct close contact with these patients suggest that nosocomial spread of influenza from staff to patients may have occurred. This is consistent with previous reports of hospital outbreaks of influenza in which HCWs were thought to have transmitted the infection to patients $(1,9,17)$ while they were incubating infection, undergoing subclinical infection or working despite the existence of mild symptoms (9). It was also possible that patients acquired the disease from visitors. However, this was less likely because the patients were all chronic inpatients and none was known to have had contact with a visitor who had influenza. The fact that seven staff members became ill after the peak outbreak of influenza in patients had
TABLE 1

Immunization and amantadine chemoprophylaxis in patients and health care workers with and without influenza symptoms

\begin{tabular}{|c|c|c|c|c|}
\hline \multirow[b]{2}{*}{$\begin{array}{l}\text { Immunization/ } \\
\text { prophylaxis }\end{array}$} & \multicolumn{2}{|c|}{ Patients } & \multicolumn{2}{|c|}{ Health care workers } \\
\hline & Influenza* & $\begin{array}{c}\text { No } \\
\text { influenza }\end{array}$ & Influenza $a^{\ddagger}$ & $\begin{array}{c}\text { No } \\
\text { influenza }\end{array}$ \\
\hline $\begin{array}{l}\text { Vaccinated } \\
\text { pre-outbreak }\end{array}$ & 1 & 2 & 3 & 4 \\
\hline $\begin{array}{l}\text { Vaccinated } \\
\text { post outbreak }\end{array}$ & 2 & 11 & 15 & 26 \\
\hline $\begin{array}{l}\text { Not vaccinated } \\
\text { Received }\end{array}$ & 13 & 4 & 1 & 2 \\
\hline amantadine & 2 & 13 & 13 & 16 \\
\hline
\end{tabular}

occurred suggests they may have become ill because of their occupational exposure. Only two of these affected HCWs acknowledged having a family member with influenza; in these two cases the direction of transmission was not clear, ie, influenza may have spread from staff to family member or vice versa.

Three of the 16 patient cases in this outbreak (18.8\%) died, a rate similar to previously documented death rates in a high risk population (16). In this geriatric population, risk factors associated with disease acquisition could be identified. A history of congestive heart failure was the only significant risk factor for influenza acquisition in this already high risk population. The fact that none of the initial patients shared a room but that $89 \%$ of these patients had congestive heart failure supports the importance of this disease as a risk factor in this population. Other previously identified risk factors (such as pulmonary disorders, diabetes, cancer and renal disorders) were not significant risk factors in this high risk population. Although considerable concern regarding patient tolerance of amantadine was expressed because of the advanced age of the patients, no adverse effects were documented.

\section{CONCLUSIONS}

The proportion of HCWs and patients who were vaccinated prior to the outbreak was low, 13 and $6 \%$, respectively. While there was no significant difference in incidence of influenza-like symptoms between those vaccinated and not vaccinated, the numbers were too small to allow conclusions. Morbidity among patient cases appeared to be high because $62.5 \%$ of cases received intravenous antibiotics. A conservative estimate of the cost of management of this influenza outbreak has been described. The cost benefit for an influenza vaccine program for HCWs has also been previously described in the literature (18). Vaccination may have prevented at least $70 \%$ of the disease morbidity suffered by this group of patients (12). The National Advisory Committee on Immunization recommends the immunization of high risk patients, as well 
as the HCWs who care for them, prior to an influenza outbreak (19). Presumably this would have prevented much of the morbidity and costs described in this report.

\section{REFERENCES}

1. Centers for Disease Control. Prevention and control of influenza: Recommendation of the immunization practices advisory committee.

Ann Intern Med 1982;101:218-22.

2. Centres for Disease Control. Prevention and control of influenza. MMWR 1987:36:373-87.

3. Kapila R, Lintz DI, Tecson FT, et al. A nosocomial outbreak of influenza A. Chest 1977:71:576-9.

4. Bauer CR, Elie K, Spence L, et al. Hongkong influenza in a neonatal unit. JAMA 1973;233:1233-5.

5. McDougal BA, Hodges GR, Lewis HD, et al. Nosocomial influenza-A infection. South Med J 1977;70:1023-4.

6. Meibalane R, Sedmak GV, Sasidharan P, et al. Outbreak of influenza in a neonatal intensive care unit. $J$ Pediatr 1977:91:974-6.

7. O'Donoghue JM, Ray CG, Terry DW, et al. Prevention of nosocomial influenza infection with amantadine. Am J Epidemiol 1973:97:276-82

8. Van Voris LP, Belshe RB, Shaffer JL. Nosocomial influenza B virus infection in the elderly. Ann Intern Med 1982;96:153-8.
9. Pachucki CT, Pappas SA, Fuller GF, et al. Influenza A among hospital personnel and patients: Implications for recognition, prevention and control. Arch Intern Med 1989;149:77-80.

10. Hammond GW, Cheang M. Absenteeism among hospital staff during an influenza epidemic: Implications for immunoprophylaxis. Can Med Assos J 1984;131:449-52.

11. Riddiough MA, Sisk JE, Bell JC. Influenza vaccination: Cost-effectiveness and public policy. JAMA 1983;249:3189-95.

12. Ruben FL. Prevention and control of influenza: Role of vaccine. Am J Med 1987;82(Suppl6A):31-4

13. Cate TR. Clinical manifestations and consequences of influenza. Am J Med 1987;82(Supp16A):15-9.

14. Barker WH, Mullooly JP. Impact of epidemic type A influenza in a defined adult population. Am J Epidemiol 1980;112:798-813.

15. Schoenbaum SC. Economic impact of influenza. Am J Med 1987;82(Suppl6A):26-30.

16. Trepaner K. Influenza outbreaks in long-term care facilities, 1988-1989 season. CDWR 1989;15-48:239-45.

17. Hoffman PC, Dixon RE. Control of influenza in the hospital. Ann Intern Med 1977:87:725-8.

18. Yassi A, Kettner J, Hammond G, Cheang M, McGill M. Effectiveness and cost-benefit of an influenza vaccination program for health care workers. Can J Infect Dis 1991:2:101-8.

19. National Advisory Committee on Immunization (NACI). Statement on influenza vaccination for the 1989-1990 season. CDWR 1989;15-23:117-22. 


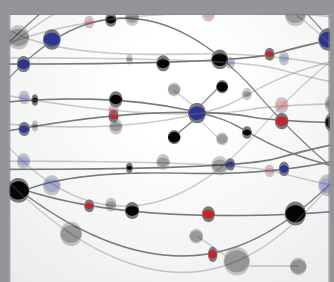

The Scientific World Journal
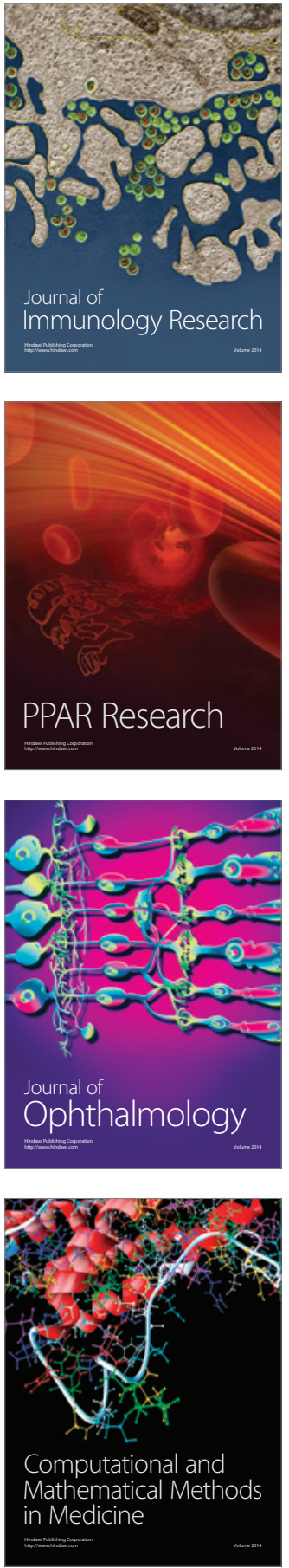

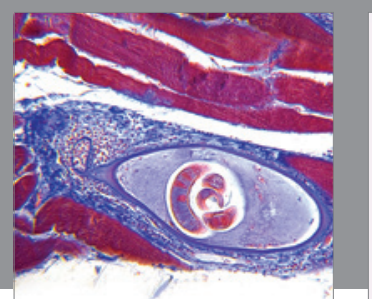

Gastroenterology Research and Practice

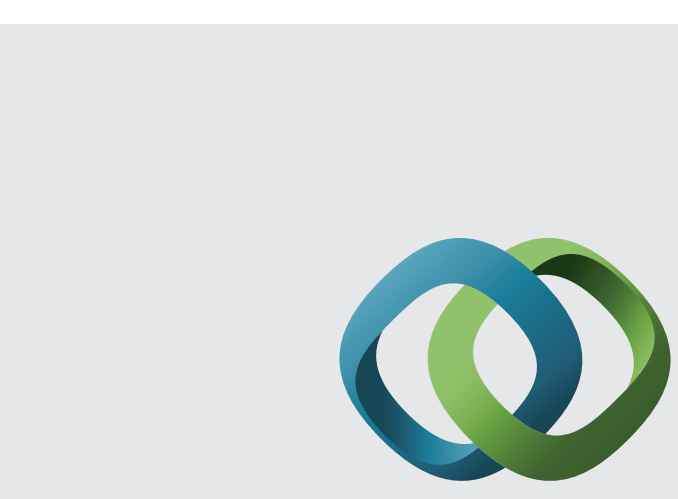

\section{Hindawi}

Submit your manuscripts at

http://www.hindawi.com
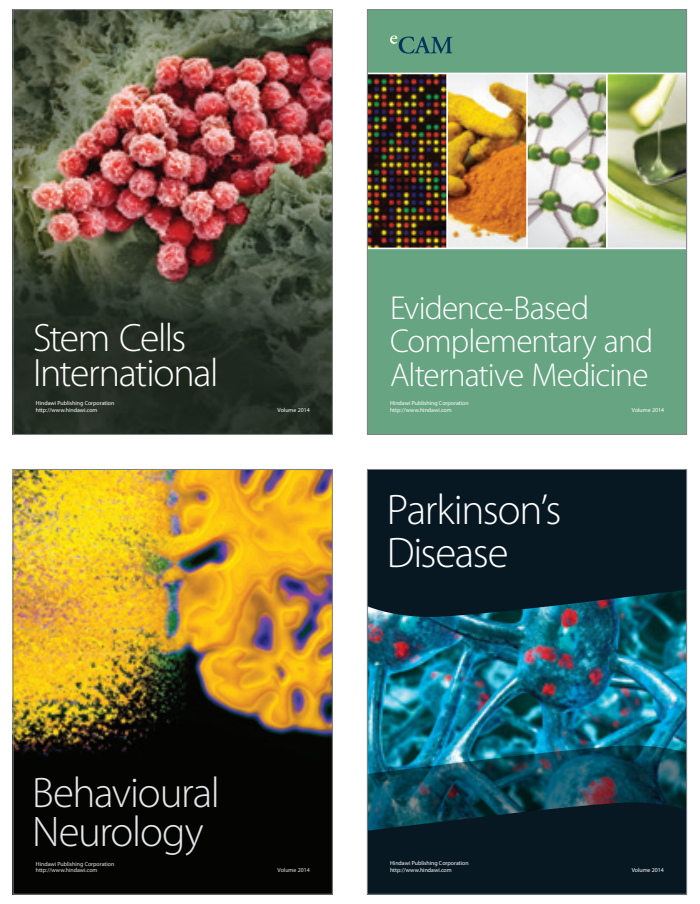
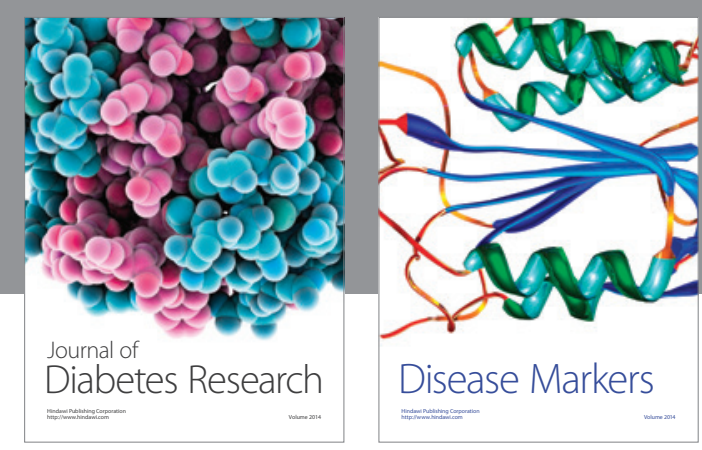

Disease Markers
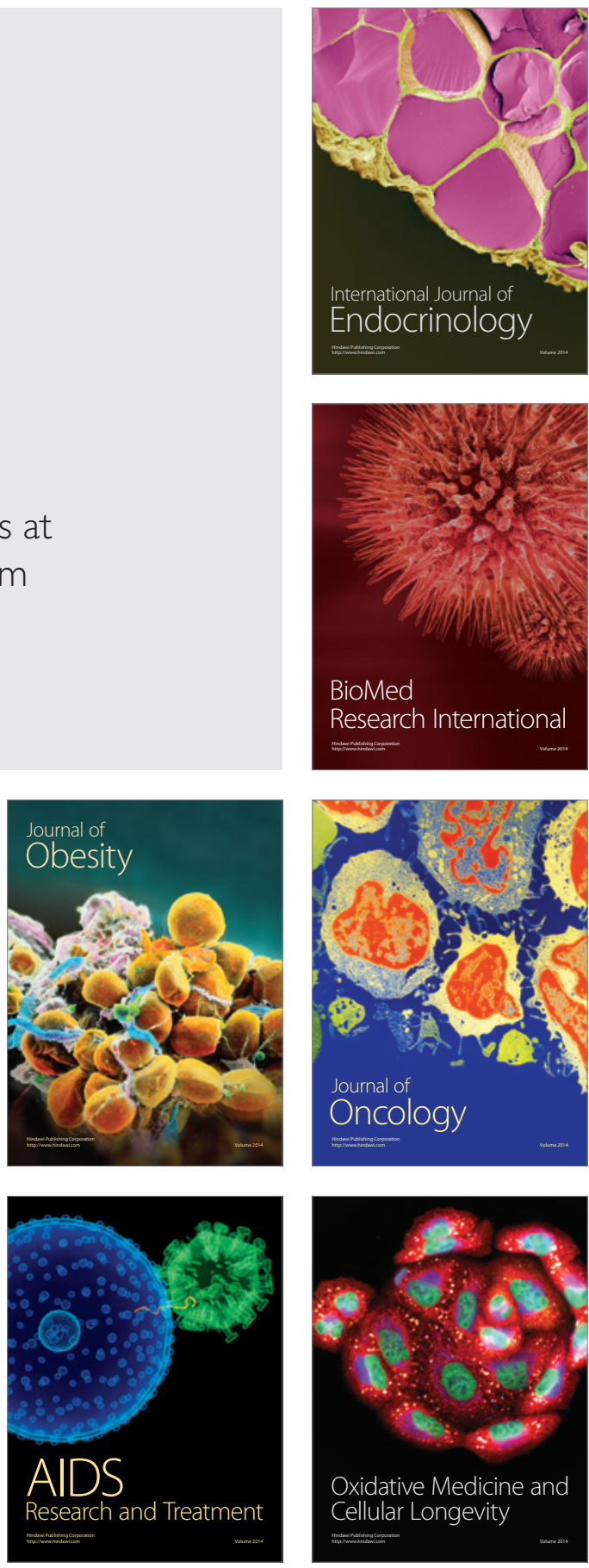